\title{
XPS and UPS Valence Band Studies of Nanocrystalline Ni-Ti Alloy Thin Films Acta Physica Polonica A 133, 613 (2018), ERRATUM
}

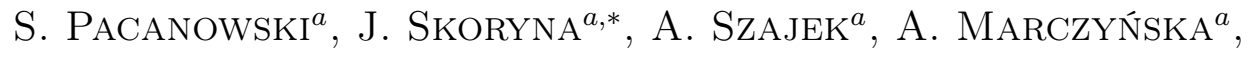 \\ H. DAWCZAK-DĘBICKI ${ }^{a, b}$, M. WERWIŃSKI ${ }^{a}$, \\ Ł. MAJCHRZYCKI ${ }^{c}$ AND L. SMARDZ ${ }^{a}$ \\ ${ }^{a}$ Institute of Molecular Physics, Polish Academy of Sciences, \\ M. Smoluchowskiego 17, 60-179 Poznań, Poland \\ ${ }^{b}$ Faculty of Physics, Adam Mickiewicz University, \\ Umultowska 85, 61-614 Poznań, Poland \\ ${ }^{c}$ Wielkopolska Centre of Advanced Technologies, Adam Mickiewicz University, \\ Umultowska 89C, 60-614 Poznań, Poland \\ orginal Doi: 10.12693/APhysPolA.133.613 \\ *e-mail: jskoryna@ifmpan.poznan.pl \\ actual Doi: 10.12693/APhysPolA.138.570

\begin{abstract}
In this contribution we study valence bands of $i n$-situ prepared nanocrystalline $\mathrm{NiTi}$ and $\mathrm{Ni}_{3} \mathrm{Ti}_{\text {alloy }}$ thin films using X-ray and ultraviolet photoelectron spectroscopy. Additionally, theoretical valence band of $\mathrm{NiTi}$ alloy was calculated by ab-initio methods. The structure and morphology of the samples were studied by X-ray diffraction and atomic force microscopy, respectively. Furthermore, hydrogen absorption and desorption kinetics at a pressure of about 1000 mbar were studied in Pd covered nanocrystalline $\mathrm{NiTi}$ alloy thin film using four-point resistivity measurements. Results showed that modifications of the valence bands of the $\mathrm{Ni}-\mathrm{Ti}$ thin films due nanocrystalline structure can influence on the room temperature hydrogen absorption and desorption kinetics.
\end{abstract}

topics: 73.22.-f, 68.55.-a, 82.80.Pv

This article was originally published on March 2018 with incorrect first two paragraphs of the introduction chapter. The correct text of the first two paragraphs of the introduction chapter is shown below:

Metallic materials based on $\mathrm{LaNi}_{5}$, TiFe, $\mathrm{ZrV}_{2}$, and FeTi alloys can reversibly absorbing hydrogen at relatively low pressure $(p<10$ bar) and temperature $(T<393 \mathrm{~K})$ and therefore were considered for hydrogen storage applications [1, 2]. In addition, the nano-structurization and alloying with other elements considerably improved the hydrogenation properties of Mg-based bulk materials [3-5].

According to [6], the hydrogenation properties of thin films should be relevant for nanostructured bulk samples. For instance, the high catalytic activity of oxidized Ni clusters was demonstrated for hydrogen absorption using Y thin films as optical indicators [7]. Importantly, the deposition process of thin films [8] is more controllable than ball-milling $[4,5]$ and as a result the role of dopants can be better determined [6]. However, due to the lattice mismatch between the film and the substrate, the dislocations and vacancies are present for higher density than in the case of bulk materials $[6,9]$.

The authors apologize for this error. 